\title{
Prevalence of coronary artery disease in a semi urban population in Southern Sri Lanka
}

\author{
J B Jayawardene ${ }^{1}$, G D N Samarutilake², M H M Zackie ${ }^{1}$, P V De Silva ${ }^{3}$, A Karunanayake \\ M A Weerasooriya ${ }^{4}$
}

(Index words: coronary artery disease, silent ischaemia, non communicable diseases, prevalence of CAD)

\begin{abstract}
Introduction Prevalence of coronary artery disease (CAD) is on the rise in Sri Lanka resulting in high hospital mortality rates. Because of paucity of community based data on CAD we carried out this study to assess prevalence of CAD and associated factors in a suburban population in Southern Sri Lanka.

Methods A cross sectional descriptive study was carried out among community living adults aged 30 years or more in Bope-Poddala Medical Officer of Health $(\mathrm{MOH})$ area. Total of 1000 eligible individuals were recruited using cluster sampling. Cardiovascular Questionnaire of London School of Hygiene was administered to each participant by trained data collectors. CAD was diagnosed using criteria by Epstein and colleagues and electrocardiograms were classified according to the Minnesota code by a Cardiologist blinded to participant details $[9,10]$.

Results Total of 579 (57.9\%) females and 421 (42.1\%) males were studied. Mean age of the group was 53 years. Among the participants $32 \%$ had cardiovascular risk related non communicable diseases (NCDs) and $22.2 \%$ had a family history of the same. Prevalence of CAD was 6.9\% (95\% $\mathrm{Cl}: 5.33-0.47)$ and silent ischaemia was $2.2 \%$ (95\% Cl: 1.29 - 3.11). Only gender and past history of cardiovascular risk related NCDs emerged as predictors of CAD.

Conclusion CAD was more prevalent among males and those with past history of cardiovascular risk related NCDs. Community based preventive programmes should be implemented to minimize the adverse impact of CAD.
\end{abstract}

Ceylon Medical Journal 2017; 62: 34-39

DOI: http://doi.org/10.4038/cmj.v62i1.8432

\section{Introduction}

Incidence of coronary artery disease (CAD) in Sri Lanka has doubled over the past two decades. According to the Annual Health Statistics 2007, incidence of CAD ranged from 645.5 to 1364.5 cases per 100,000. It was the leading cause of hospital deaths in the country during the last 10 years accounting for nearly $10 \%$ of all hospital deaths [3]. However, net death rate due to CAD events reduced drastically in Western countries due to technically advanced interventional procedures [1,2]. These treatment modalities are expensive and in Sri Lanka these patients have to bear a substantial portion of the expenditure despite government subsidies.

According to the American Heart Association (AHA) statistics, the prevalence of CAD in the age group 20-39 years is $0.6 \%$ in both sexes. But a higher prevalence is seen in the age group 40-59 years in both males $6.3 \%$ and females $5.6 \%$. Prevalence of CAD increases with advancing age. Nearly $20 \%$ of males and $9.7 \%$ of females in the age group of $60-79$ years and $32 \%$ of males and $19 \%$ of females in the age group more than 80 years [4]. Prevalence of CAD in American population during $1971-75$ was $5.1 \%$. This showed an upward trend during the period 1988-1994 leading to $10.4 \%$ pre-valence. However, this rate dramatically reduced to 7.6\%between 1999-2006 due to success of community interventions. Risk of CAD is associated with hyper-tension, elevated serum cholesterol, diabetes mellitus and smoking [4].

Aljefree and Ahemed reported that the prevalence of cardio vascular diseases (CVD) in Saudi Arabia is 5.5\% and hypertension, diabetes mellitus (DM), obesity, smoking and poor dietary habits were risk factors for the CVD in the Gulf region [5]. Sarvotham and Berry studied the prevalence of coronary heart disease (CHD)

${ }^{1}$ Cardiology Unit, National Hospital of Sri Lanka, ${ }^{2}$ Ministry of Health, ${ }^{3}$ Faculty of Medicine, University of Ruhuna, ${ }^{4}$ Teaching Hospital Karapitiya, Sri Lanka.

Correspondence: JBJ, e-mail: <jayj426000@yahoo.com>. Received 7 September 2016 and revised version accepted 14 December 2016.

This is an open-access article distributed under the terms of the Creative Commons Attribution License, which permits unrestricted use, distribution, and reproduction in any medium, provided the original author and source are credited. 
in an urban population of India applying the case definitions published by Epstein and associates. Electrocardiograms were classified according to the Minnesota code. Prevalence of probable cases (P1 and P2) among males was 65.4/1000 population and among females 67.2/1000 population. Prevalence of suspected cases among males was 38.8/1000 population and in females 115.1/1000 population [6].

In 2005 in Sri Lanka, age and sex standardized prevalence of ischaemic heart disease (IHD) was estimated as 9.3\% [15]. In this study, cardiac ischaemia was diagnosed on ECG evidence or positive Rose Angina Questionnaire (RAQ). Hypertension, dyslipidaemia, smoking, physical inactivity, obesity, diabetes mellitus and family history of IHD were identified as risk factors of CAD. A similar study was done in India using RAQ and Minnesota ECG coding. In this study, crude and age adjusted prevalence of CAD was reported as 5.8\% and $3.8 \%$ respectively [17].

A comparative study was conducted to assess the prevalence of macro vascular diseases in non insulin dependent diabetics. In this study significantly higher prevalence of ECG coronary possible (on Minnesota coding) was detected in the study group compared to the age and sex matched control group [16]. Prevalence of CAD was assessed using the London School of Hygiene questionnaire and 12 lead ECG to diagnose definitive CAD (positive symptoms and ECG), evidence of CAD (history alone) and silent ischaemia (positive ECG without symptoms). According to the results of this study prevalence of definitive CAD was 16/1000 (95\% CI 9-27/1000), evidence of CAD was 54/1000 (95\% CI: 40-71/1000) and silent CAD was 32/1000 (21-46/ 1000). Mendis and colleagues reported that hypertension, hyperlipidaemia, high BMI and current smoking were significant predictors of CAD [13]. Occurrences of cardiovascular risk factors were assessed in a sample of male patients who were awaiting coronary artery bypass grafting (CABG) in a provincial hospital in Sri Lanka. This study reported prevalence of diabetes 39.1\%, dyslipidaemia 35.5\%, hypertension 47.5\%, smoking $74.5 \%$ and alcohol use $75.9 \%$. However, only diabetes and hypertension emerged as significant predictors of disease severity [14].

Few community studies have been done on prevalence of coronary artery disease (CAD) in Sri Lanka. Community studies could yield valuable information about the extent and nature of the problem. Such information would be of value in designing a national health programme for the prevention of CAD in Sri Lanka.

The objectives of this study were to determine the prevalence and risk factors of CAD in a semi urban population in southern Sri Lanka.

\section{Methodology}

A community based cross sectional descriptive study was carried out. Bope-Poddala $\mathrm{MOH}$ area was selected as it represented the semi urban population in the Galle District. The study was conducted from May 2011 to April 2012. Subjects were selected using cluster sampling. All persons age $>30$ years living in the selected $\mathrm{MOH}$ area were considered as the study population. However, people living temporarily in the area and persons who were extremely ill or persons aged $\geq 60$ years who were physically and psychologically unsound to be recruited to the study were excluded.

Sample size was calculated to detect a prevalence of $1.4 \%$ at a precision of 0.01 [3,7]. Since this is a community based study, it was decided to employ cluster sampling method based on public health midwife (PHM) areas. Design effect was taken as 2 . Calculated sample size was 963 with an anticipated $10 \%$ non response rate.

Cluster size was taken as 30 and the sample size was rounded up to form 33 clusters. Theses clusters were distributed among the PHM areas according to the proportions of population above 30 years. Study units in each cluster were selected randomly using the updated voters list of the respective Grama Niladhari (GN) division as the sampling frame. Only one person from each household was selected for the study. If the selected person was not contacted after two consecutive visits it was considered as a non response, and the next available eligible person was recruited. An additional 10 participants were recruited randomly to round up the sample size to 1000 study units. All participants were subjected to electrocardiogram (ECG). ECGs were analysed by a cardiologist who was blinded to clinical data according to the Minnesota code without the prior knowledge on participants [10]. Persons with clinical or electrocardio-graphic evidence of CAD were advised and referred for appropriate medical care.

\section{Data collection tool}

An interviewer administered questionnaire was used to obtain the socio demographic and clinical data. Weight, height and blood pressure were measured. A modified version of the London School of Hygiene Cardiovascular Questionnaire was administered [8].

All study subjects had a basic medical examination and a resting 12 lead electrocardiogram (ECG). ECG is the most important first line investigation in diagnosis of CAD and plays a major role in the diagnosis silent ischaemia. The diagnosis of CAD was based on criteria laid down by Epstein and associates [9].

Participants without symptoms but with electrocardiographic ischemic changes were categorized as having silent ischaemia. Silent ischaemia was further categorized according to the Minnesota code [10]. 
Persons diagnose with CAD were referred to the nearest hospital with a cardiology unit. Persons with elevated blood pressure without CAD were advised to go to a local physician for confirmation of hypertension and follow up treatment. Relevant health advice was given to all participants.

Written informed consent was obtained from all eligible participants prior to enrolment in the study. Ethics clearance was obtained from the Ethics Review Committee, Faculty of Medicine, University of Ruhuna.

Binary logistic regression model was developed to assess associations with CAD. Variables entered in the logistic regression model were age, gender, BMI group, smoking, past history of NCD, past history of hypertension, family history of NCD and occupation for modeling.

\section{Results}

Non response rate was $18.2 \%$. Total of 1000 study subjects were recruited. Majority of the study population comprised of females $(n=579,57.9 \%)(42.1 \%)$ (Table 1).

\section{Table 1. Socio-demographic characteristics} according to gender

\begin{tabular}{|c|c|c|c|}
\hline & Female & Male & Total \\
\hline \multicolumn{4}{|l|}{ Age groups (Years) } \\
\hline $30-39$ & $101(17.4)$ & $92(21.9)$ & 193(19.3) \\
\hline $40-49$ & 138(23.8) & $92(21.9)$ & $230(23.0)$ \\
\hline $50-59$ & $147(25.4)$ & $95(22.6)$ & $242(24.2)$ \\
\hline $60-69$ & $111(19.2)$ & $75(17.8)$ & 186(18.6) \\
\hline$\geq 70$ & $82(14.2)$ & $67(15.9)$ & $149(14.9)$ \\
\hline Total & $579(100.0)$ & $421(100)$ & $1000(100.0)$ \\
\hline \multicolumn{4}{|l|}{ Marital status } \\
\hline Married & $543(93.8)$ & $389(92.4)$ & $931(93.1)$ \\
\hline Never married & $36(6.2)$ & $32(7.6)$ & $68(6.8)$ \\
\hline Total & $579(100.0)$ & $421(100.0)$ & $1000(100.0)$ \\
\hline \multicolumn{4}{|l|}{ Occupation } \\
\hline Professional -Higher & $0(0.0)$ & $3(0.7)$ & $3(0.3)$ \\
\hline Professional -Lower & $3(0.3)$ & $24(5.7)$ & $27(2.7)$ \\
\hline Skilled & $42(7.3)$ & 133(31.6) & $175(17.5)$ \\
\hline Semi skilled & $27(4.7)$ & $123(29.2)$ & $150(15.0)$ \\
\hline Manual & $507(87.6)$ & $138(32.8)$ & $645(64.5)$ \\
\hline Total & $579(100.0)$ & $421(100.0)$ & $1000(100.0)$ \\
\hline \multicolumn{4}{|l|}{ Smoking status } \\
\hline Never smoked & $573(99.0)$ & $221(52.5)$ & $794(79.4)$ \\
\hline Current & $2(0.3)$ & $113(26.8)$ & $115(11.5)$ \\
\hline Ex smoker & $4(0.7)$ & $87(20.7)$ & $91(9.1)$ \\
\hline Total & $579(100.0)$ & $421(100.0)$ & $1000(100.0)$ \\
\hline \multicolumn{4}{|l|}{ Alcohol use } \\
\hline Never & 576(99.5) & $295(70.1)$ & $871(87.1)$ \\
\hline User & $3(0.5)$ & $126(29.9)$ & $129(12.9)$ \\
\hline Total & 579 (100.0) & $421(100.0)$ & $1000(100.0)$ \\
\hline
\end{tabular}

Mean age of participants was 53 years (SD - 13.76). Nearly $80 \%$ of the sample were aged $>40$ years and $34 \%$ of the participants were aged $\geq 60$. More than $90 \%$ of both males and females were married. Majority were manual labourers. House wives were classified as manual workers. Majority of the professionals were males. Nearly $80 \%$ of the population were non smokers, $21 \%$ of males were ex-smokers and $27 \%$ were current smokers. Prevalence of smoking was extremely low among females. Prevalence of alcohol use in the total population was $13 \%$ and prevalence among males was $29.9 \%$.

Thirty two percent had a past history of CVD $(n=320)$ and $22 \%$ had a family history (Table 2). Nearly $16 \%$ of the participants had a history of hypertension, 8.3\% had a past history of DM. Mean BMI was 22.6 $(\mathrm{SD}=4.3)$. Majority (58\%) had a normal BMI.

Mean systolic blood pressure was $133.6 \mathrm{mmHg}$ $(\mathrm{SD}=19.2)$ and median was $130 \mathrm{mmHg}$ range 84-200 mmHg. Mean diastolic pressure was $80.7 \mathrm{mmHg}$ (SD $=11.9$ ) and the median was $80 \mathrm{mmHg}$. However, neither systolic nor diastolic pressures were associated with CAD status.

Three hundred and two (30.2\%) had a history of chest pain. Sixty nine of them were electrocardiographically positive and was diagnosed with CAD. Prevalence of CAD was 6.9\% (95\% CI 5.33-8.47). Crude rate of CAD was 7.01\% (95\% CI 6.94-7.08) and age and sex standardized prevalence was 6.6\%. Age standardized prevalence of CAD for the males was $8.2 \%$ and that of the females was $5.2 \%$.

Silent ischaemia was observed among 22 individuals who had no chest pain but were electrocardiographically positive. Therefore, the prevalence of silent ischaemia in this community was $2.2 \%$.

Males had higher prevalence of CAD (8.8\%) compared to females (5.5\%) (Table 4). Prevalence of CAD increased with advancing ages. The highest prevalence $(12.9 \%)$ of CAD was observed in the age group 60-69 years.

Prevalence of CAD was; high BMI group 9.8\%, normal BMI group 5.3\% and low BMI group 8.3\%. Skilled workers had the highest prevalence of CAD (10.3\%). These differences were not statistically significant.

Prevalence of CAD among ex-smokers (12.1\%) is almost 2 times higher compared to that in the current (6.1\%) and non smokers (6.7\%). The prevalence of CAD was higher among current alcohol users (8.5\%) than in non-users $(6.7 \%)$. These differences were not statistically significant.

Higher prevalence of CAD (10.9\%) was observed in those with past history of cardiovascular related NCDs.

Prevalence of P1 increased with age except in the $\geq 70$ year age group. 
Table 2. Health status of study population

\begin{tabular}{|c|c|c|c|}
\hline & Female & Male & Total \\
\hline \multicolumn{4}{|l|}{ Past History of CVD } \\
\hline Yes & $189(32.6)$ & $131(31.1)$ & $320(32.0)$ \\
\hline No & $390(67.4)$ & $290(68.9)$ & $680(68.0)$ \\
\hline Total & $579(100.0)$ & $421(100.0)$ & $1000(100.0)$ \\
\hline \multicolumn{4}{|l|}{ Family History of CVD } \\
\hline Yes & $140(24.2)$ & $82(19.5)$ & $222(22.2)$ \\
\hline No & $439(75.8)$ & $339(80.5)$ & $778(77.8)$ \\
\hline Total & $579(100.0)$ & $421(100.0)$ & $1000(100.0)$ \\
\hline \multicolumn{4}{|l|}{ Past History of } \\
\hline \multicolumn{4}{|l|}{ Hypertension } \\
\hline Yes & $101(17.4)$ & $58(13.8)$ & $159(15.9)$ \\
\hline No & $478(82.6)$ & $363(86.2)$ & $841(84.1)$ \\
\hline Total & $579(100.0)$ & $421(100.0)$ & $1000(100.0)$ \\
\hline \multicolumn{4}{|l|}{ Past History } \\
\hline \multicolumn{4}{|l|}{ of Diabetes } \\
\hline Yes & $41(7.1)$ & $42(10.0)$ & $83(8.3)$ \\
\hline No & $538(92.9)$ & $379(90.0)$ & $917(91.7)$ \\
\hline Total & $579(100.0)$ & $421(100.0)$ & $1000(100.0)$ \\
\hline \multicolumn{4}{|l|}{ BMI(Body } \\
\hline \multicolumn{4}{|l|}{ Mass Index) } \\
\hline $\operatorname{Low}\left(>18 \mathrm{Kg} / \mathrm{m}^{2}\right)$ & $81(14.2)$ & 63(15.3) & $144(14.7)$ \\
\hline Normal (18-25 Kg/m²) & $334(58.5)$ & 238(57.9) & $572(58.2)$ \\
\hline $\operatorname{High}\left(\geq 25 \mathrm{Kg} / \mathrm{m}^{2}\right)$ & 156(27.3) & $110(26.8)$ & 266(27.1) \\
\hline Total & $571(100.0)$ & $411(100.0)$ & $982(100.0)$ \\
\hline
\end{tabular}

Table 3. Prevalence of coronary artery disease (CAD)

\begin{tabular}{lcc}
\hline CAD status & Prevalence & $95 \%$ CI* \\
\hline CAD & $6.9 \%$ & $5.33-8.47$ \\
Crude CAD & $7.01 \%$ & $6.94-7.08$ \\
& & \\
Age and sex standardized CAD & $6.6 \%$ & $6.54-6.66$ \\
Age standardized CAD in males & $8.2 \%$ & $8.09-8.31$ \\
Age standardized CAD in females & $5.2 \%$ & $5.12-5.28$ \\
CAD sub types & & \\
Suspected (S) & & \\
Probable 1(P1) & $1.2 \%$ & $0.53-1.87$ \\
Probable 2(P2) & $5.0 \%$ & $3.65-6.35$ \\
Silent ischaemia & $0.7 \%$ & $0.18-1.22$ \\
\end{tabular}

Low BMI females had higher prevalence of P2 and silent ischaemia. Normal BMI females showed the highest prevalence of P1.

Odds of having CAD in males was 1.7 times that in females (Adjusted OR=1.68, 95\% CI 1.03-3.02; $p=0.02$ ). Odds of having CAD in those with a past history of NCDs were 2.4 times than those without
(Adjusted OR=2.36, 95\% CI 1.4 - 3.9; $p=0.11$ ). Age ( $p=0.32$ ), BMI ( $p=0.61)$, smoking ( $p=0.26$ ), past history of hyper-tension $(p=0.44)$ and family history of NCD $(p=0.36)$ were not significantly associated with CAD.

Odd of having silent ischaemia was 3 times higher (adjusted OR=3.05, 95\% CI 1.27-7.95; $p=0.022$ ) in the hypertensive compared to the normotensive participants. Gender $(p=0.215)$, past history of diabetes $(p=0.178)$ and obesity or high BMI $(p=0.219)$ did not have significant associations with silent ischaemia.

\section{Discussion}

The prevalence of CAD in Bope-Poddala $\mathrm{MOH}$ area was $6.9 \%$. The prevalence of suspected cases was $1.2 \%$ and the prevalence of probable cases of $\mathrm{P} 1$ was $5 \%$ and P2 was $0.7 \%$. The prevalence of silent ischaemia was $2.2 \%$. P1 were the most prevalent.

These findings could be generalized to suburban populations in Galle district as the characteristics of people in the selected $\mathrm{MOH}$ area was representative of such populations. Over representation of females may have occurred due to sampling procedure adopted to overcome the non responses. Proportion of females in the Galle district was 53.7\% [18]. Therefore, effects of female overrepresentation could be considered minimal. Past history NCDs and gender emerged as significant predictors of CAD.

Comparison with prevalence rates in other studies is difficult due to differences in diagnostic criteria. However, the prevalence rates do not show significant deviations from other studies.

Prevalence of CAD was analysed by population subgroups to assess the variations. Males had higher prevalence of CAD compared to females. Females did not have higher prevalence of $\mathrm{S}$ or P2. These observations indicate that the borderline ECG changes and T inversions which are commoner in women had not influenced the results of the study.

Higher rates of smoking and alcohol use could account for higher prevalence of CAD in males. Nearly $30 \%$ of males were alcohol users and prevalence of smoking among males was 26.8\%. De Silva et al reported that in 2009 prevalence of alcohol use among males was $32.9 \%$ and the prevalence of smoking was $29.9 \%$ in urban areas and $24.4 \%$ in rural areas [12]. Pathmeswarn et al reported that in 1997 prevalence of alcohol use among the males as 33.3\%. Prevalence of smoking and alcohol use in our study is similar to that reported previously.

Prevalence of P1 increased with age. This is similar to results published by The American Heart Association and other studies [4-6]. However, one study reported higher rates of CAD in females [6]. Timing of the study, geographical differences and cultural differences could have contributed to this difference. 


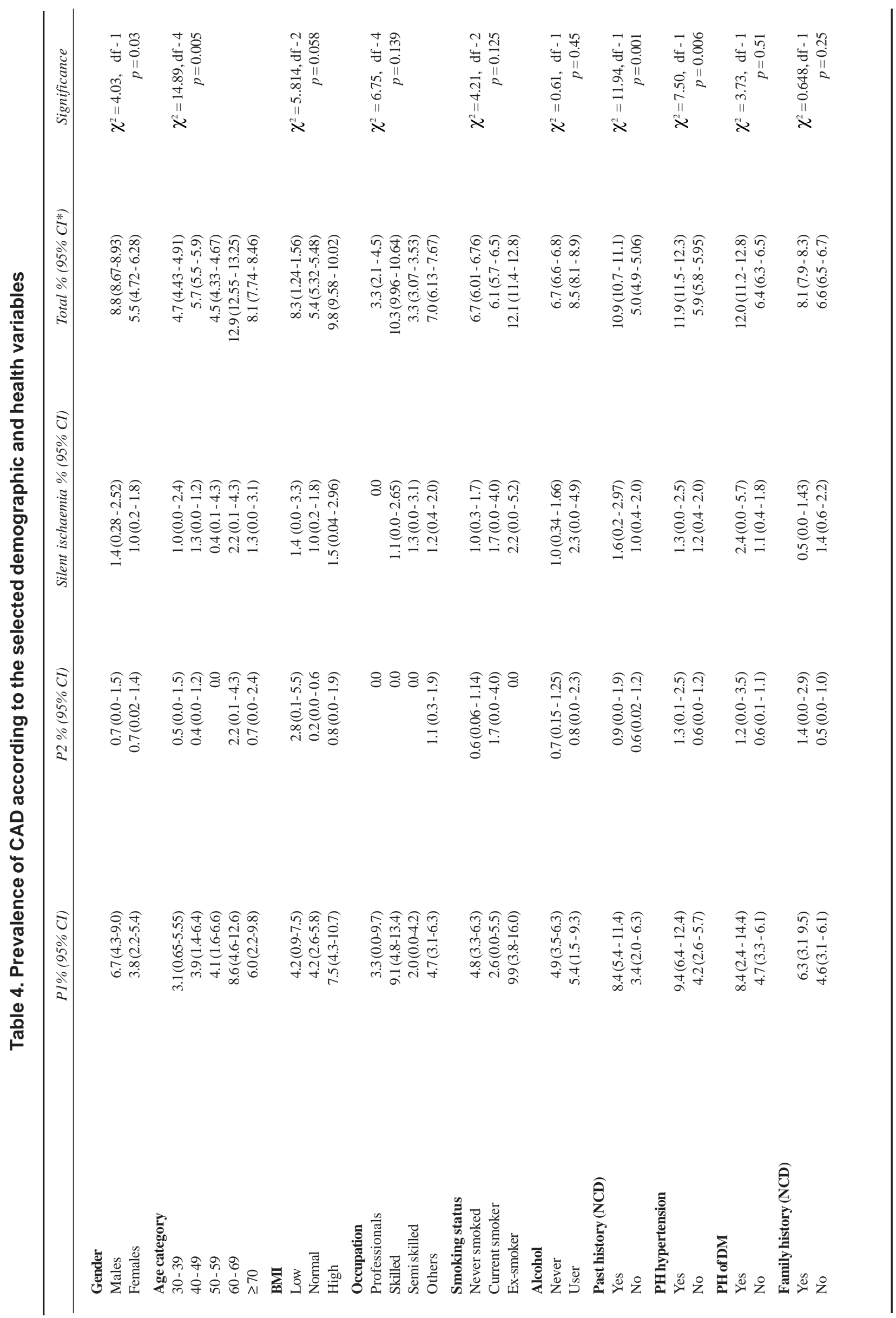


Table 5. Prevalence of CAD by the gender and age group

\begin{tabular}{lrrrr}
\hline Gender & Age group & & & \\
& & $P 1(\%)$ & $P 2(\%)$ & $S(\%)$ \\
\hline Female & $30-39$ & $2(2.0)$ & $0(0.0)$ & $1(1.0)$ \\
& $40-49$ & $4(2.9)$ & $1(0.7)$ & $2(1.4)$ \\
& $50-59$ & $6(4.1)$ & $0(0.0)$ & $0(0.0)$ \\
& $60-69$ & $8(7.2)$ & $2(1.8)$ & $2(1.8)$ \\
Male & $\geq 70$ & $2(2.4)$ & $0(0.0)$ & $1(1.2)$ \\
& & & & \\
& $30-39$ & $4(4.3)$ & $1(1.1)$ & $1(1.1)$ \\
& $40-49$ & $5(5.4)$ & $0(0.0)$ & $1(1.1)$ \\
& $50-59$ & $4(4.2)$ & $1(1.1)$ & $1(1.1)$ \\
& $60-69$ & $8(10.7)$ & $1(1.3)$ & $2(2.7)$ \\
& $\geq 70$ & $7(10.4)$ & $1(1.5)$ & $1(1.5)$ \\
\hline
\end{tabular}

Prevalence of CAD among those with a past history of NCDs was nearly $11 \%$. Gender and past history were significant predictors of CAD. These findings are consistence with findings of other studies done in Sri Lanka and in the region [13,14,15,16,17].

Gender and cardiovascular risk related NCDs, should be considered risk factors when screening for CAD. Past history of hypertension had a significant association with silent ischaemia. Based on the findings we recommend health education for high risk groups and programmes aimed at preventing NCDs. Standardizing community screening method to identify CAD should be developed and prevalence of CAD should be estimated using such methods in a nationally representative sample.

\section{Conflicts of interest}

There are no conflicts of interest.

\section{References}

1. Bonow RO, Douglas LM, Douglas PZ, Libby P. Branwald's Heart Disease, A Textbook of Cardiovascular Medicine, 9th Edn., Saunders, Missouri, 2012.

2. Kartists DG, Gersh BJ, Camm JA. Clinical Cardiology, Current Practice Guidelines 1st Edn., Oxford University Press, Oxford, 2013.

3. Department of Health Services, Annual Health Statistics 2007. Colombo, Sri Lanka, 2007

4. Mazaffarian D, Benjamin EJ, Go AS, et al. American Heart Association, National Health and Nutrition Survey 2009-2012. Circulation 2015; 131: e29 - e322.
5. Aljefree N, Ahmed F. Prevalence of Cardiovascular Diseases and Associated Risk Factors among Adult Populations in Gulf Region: A Systematic Review. Advances in Public Health, vol. 2015, Article ID 235101, 23 pages, 2015. doi:10.1155/ 2015/235101.

6. Sarvotham SG, Berry JN. Prevalence of Coronary Heart Disease in an Urban Population in Northern India. Circulation 1968; 37: 939-52.

7. Lwanga SK, Lemeshow S. Sample size determination in health studies: a practical manual, World Health Organization, Geneva 1991.

8. London School of Hygiene - cardiovascular questionnaire. Available from www.cuore.iss.it/eurociss/indaginequestionari_LSHTM.pdf

9. Epstein FH Ostrander LD Jr, Johnson BC, et al. Epidemiological studies of cardiovascular disease in a total community - Tecumesh. Michigan. Ann Intern Med 1965; 62: 1170.

10. Blackburn H, Keys A, Simpson F, et al. Electrocardiogram in population studies: Classification System, Circulation 1960; 21: 1160 .

11. Somatunga LC, Ratnayake LVR, Wijesinghe WMDNK, Yapa YMMM, Cooray MPNS. National alcohol use prevalence survey. Journal of Post Graduate Institute of Medicine 2014; 1: E7: 1-12.

12. De Silva V, Samarasingh, D, Gunawardena N. Alcohol and tobacco use in two districts in Sri Lanka. Ceylon Med J 2009; 54: 119-24.

13. Mendis S,Ekanayake EM. Prevalence of coronary heart disease and cardiovascular risk factors in middle aged males in a defined population in Sri Lanka. Int J Cardiol 1994; 46: 135-42.

14. Wicramathilake CM, Withanawasam BPS, Mohideen MR. Cardiovascular risk factors, socioeconomic determinants and angiographic severity of coronary artery disease in patients awaiting coronary artery bypass grafting in a provincial hospital Sri Lanka. Galle Medical Journal 2014; 19: 12-7.

15. Katulanda P, Liyanage IK, Caldera R, Constantine GR, Sheriff $\mathrm{R}$, Mathews D. Prevalence of ischaemic heart disease and its risk factors in Sri Lanka. Ceylon Med J 2010; 55: 53.

16. Fernando DJ, Siribaddana S, Perera N, Perera S, de Silva D. The prevalence of macrovascular disease and lipid abnormalities amongst diabetic patients in Sri Lanka. Postgraduate Medical Journal 1993; 69: 557-61.

17. Krishnan MN, Zachariah G, Venugopal K, et al. Prevalence of coronary artery disease and its risk factors in Kerala, South India: a community-based cross-sectional study. BMC Cardiovascular Disorders 2016; 16: 12. doi: 10.1186/s12872016-0189-3.

18. Department of Census and Statistics. Census of Population and Housing 2012. Department of Census and Statistics, Colombo 2012. 